\title{
Ultrafast optics with slow electrons
}

\author{
Nahid Talebi ${ }^{1, *}$ and Christoph Lienau ${ }^{2}$ \\ ${ }^{1}$ Stuttgart Center for Electron Microscopy, Max Planck Institute for Solid State Research, 70569 \\ Stuttgart, Germany \\ ${ }^{2}$ Institute of Physics and Center of Interface Science, Carl von Ossietzky University, 26129 Oldenburg, \\ Germany
}

\begin{abstract}
We study the interaction of swift electrons with laser and solids using a numerical approach, and specifically show that low-energy electrons can be used to map the time-resolved response of a Fermi gas.
\end{abstract}

\section{Introduction}

Point-projection electron microscopes (PPMs) have been introduced as compact setups for imaging samples with high spatial resolution, especially when holography is considered [1]. By introducing sharp metallic tips as an efficient near-point photoemission electron source [2, 3], PPMs have been advanced to include ultrashort electron wave packets. Such electron pulses can in principle be used to investigate the ultrafast responses of the samples, though this yet has to be explored.

Here, we study the interaction of low-energy electron wave packets with laser beams and nanostructures, using a numerical approach based on a combined system of Maxwell and Schrödinger equations [4]. A carbon nanorod is considered as the sample, which is illuminated with a 20-fs laser pulse at a center wavelength of $1250 \mathrm{~nm}$, and the excitations are probed with an electron wave packet at the energy of $60 \mathrm{eV}$ (Fig. 1a). The sample was modelled with a dielectric function and imbedded in a step cylindrical potential at the height of the work function of the carbon $(5 \mathrm{eV})$. The scattered field (Fig. $1 b)$ is used to calculate the electromagnetic potentials, which are inserted in the timedependent Hamiltonian to simulate the evolution of the electron wave packet.

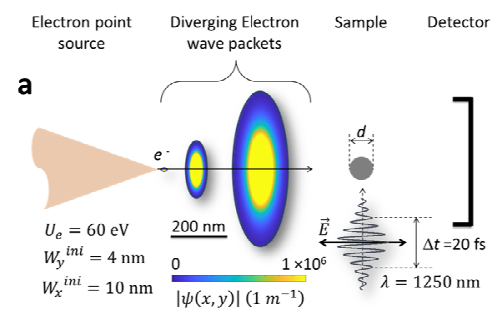

b

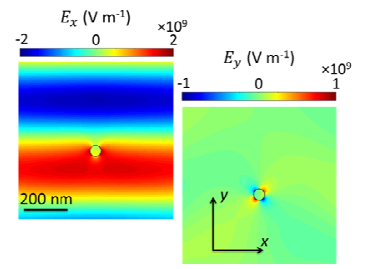

Fig. 1. (a) The topology of the system, constituting of a low-energy electron wave packet interacting with a carbon nanorod which is illuminated by a $20-\mathrm{fs}$ laser pulse at $1250 \mathrm{~nm}$. (b) The carbon nanorod is modeled by the dielectric function of carbon and the scattered field and the electromagnetic potentials are calculated. Shown are the spatial distributions of the total $x$ - and $y$ components of the electric field, at a given time. 


\section{Results and discussions}

The probe electron wave packet has an initial energy of $60 \mathrm{eV}$. The electron source is located at the distance of $1 \mu \mathrm{m}$ from the sample and emits an electron wave packet with lateral and longitudinal broadenings of $4 \mathrm{~nm}$ and $10 \mathrm{~nm}$, respectively. The resulting wave packet expands via propagation in vacuum and approaches the sample after approximately $200 \mathrm{fs}$. The temporal delay between the laser peak and the electron wave packet upon their arrival at the sample is set to $\delta \tau=0$. The time of travel of the electron from the source to the sample allows for a reasonable expansion in the lateral direction, so the electron effectively resembles a plane wave. The peak intensity and temporal broadening of the laser beam is $26 \mathrm{TW} \mathrm{cm}^{-2}$ and $20 \mathrm{fs}$, respectively. The interaction of the laser beam with the dielectric function of the nanotube drives a strong optical nearfield in the vicinity of the tube which accelerates the probe electrons. In addition couples to the free electrons of the sample, initiates the photoemission process, and causes an isotropic propagation of the photoemission electrons at $0.4 \%$ of the velocity of light, with the kinetic-energy distribution of only $0.1 \mathrm{eV}$ (Figs. 2a and b). The photoemission electrons are accelerated by the nearfield distribution in a quite fast process, and will not experience a quiver motion back towards the surface, thanks to the high field intensity introduced here. The interaction of the electron probe with the laser and sample is surprisingly complex and launches a variety of different physical processes, including: $1^{\text {st }}$-scattering of the electrons to a homogeneous cloud surrounding the particle and propagating at a velocity much lower than the velocity of the probe electron. This second cloud of scattered electrons forms an elliptical isotime curve in the momentum space, in contrast with the first bunch of photoemission electrons which are more or less distributed in a circular and isotropic isotime curve. $2^{\text {nd }}$ - lateral interferences which is the basis for inline electron holography, $3^{\text {rd }}$ - several energy loss and gain peaks, at the harmonics of the laser energy as well as harmonics of the work function, $4^{\text {th }}$ - appearance of several Ewald spheres displaced by an amount of energy equal to the work function, $5^{\text {th }}$ - asymmetric loss and gain peaks, which is representative for asymmetric transition rates for loss and gain channels dissimilar to the results which are obtained using perturbation analysis $[5,6]$, and finally $6^{\text {th }}$ - Transverse interference patterns are well resolved, accounting for interference between the scattered electrons by the sample and the incident beam (Fig. 3). Clearly, the interaction with the near-field is confined to a thin rim around the nanotube due to the high longitudinal momentum of the probe electrons. Additionally, the angular range of the scattered electrons is very much energy dependent. Several domains are distinguishable, including focused electron regions which consist of forwardly scattered beams which are energetically distributed from 40 $\mathrm{eV}$ to $90 \mathrm{eV}$, and heavily scattered electron wave packets which constitute a narrower energy range. Most interestingly, and of high relevance for future experiments, the lateral interference pattern is also strongly influenced by the laser excitation.

As a summary, our results indicate the promise of slow-electron point-projection electron microscopy for probing the ultrafast oscillations of Fermi electron gases and liquids, as well as ultrafast optical processes in solids. In addition, angle resolved energyloss and -gain spectroscopy of slow electrons interacting with nanostructured samples unravels a multitude of physical processes and might be used to examine interferences between different quantum pathways involved in the experiments. 


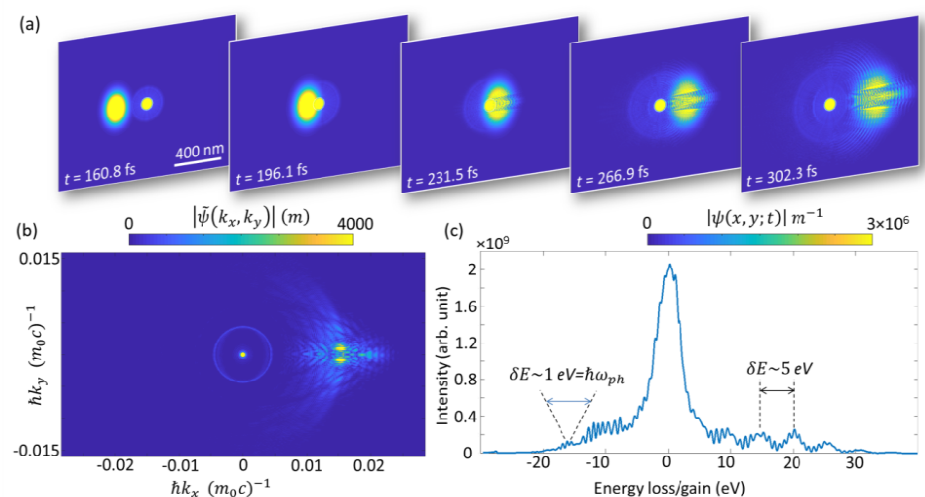

Fig. 2. (a) Snapshots of the dynamics of a low-energy electron wave function during its interaction with the laser-triggered oscillations of Fermi electrons in a carbon nanorod, (b) The momentum-space representation of the total wave function, after interaction of the electron probe with the sample, (c) The total energy distribution of the electron integrated over the lateral direction, after the interaction.

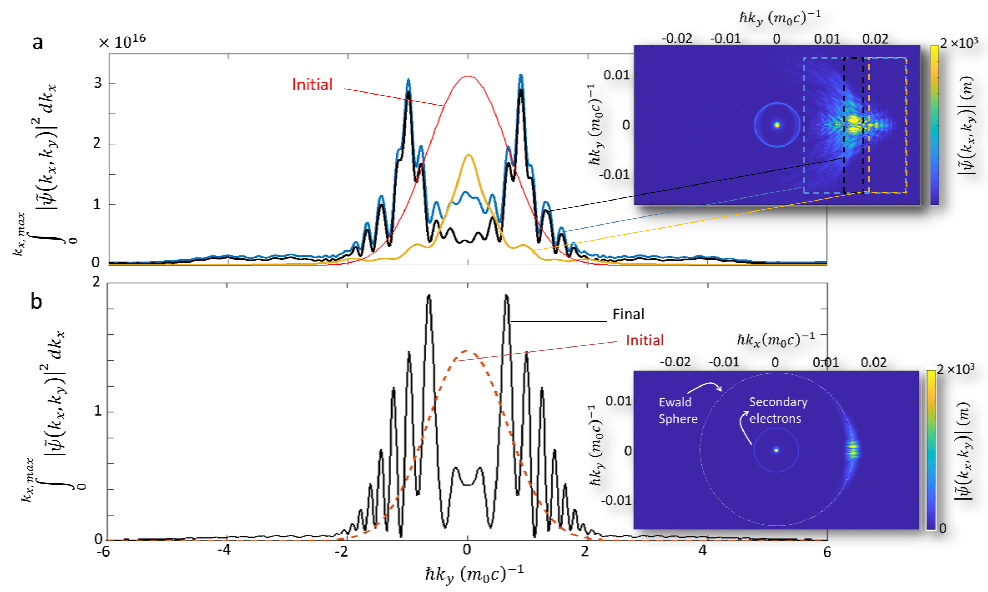

Fig. 3. The lateral distribution of the electron probability integrated over the longitudinal momentum ranges displaced at the inset, for the sample (a) illuminated, and (b) not excited with the laser.

\section{References}

1. H. W. Fink, W. Stocker, and H. Schmid, Phys. Rev. Lett. 65, 1204 (1990).

2. P. Gross, M. Esmann, S. F. Becker, J. Vogelsang, N. Talebi, and C. Lienau, Adv. Phys. :X 1, 297 (2016).

3. J. Vogelsang, J. Robin, B. J. Nagy, P. Dombi, D. Rosenkranz, M. Schiek, et al., Nano Lett. 15, $4685(2015)$.

4. N. Talebi, New J. Phys. 18, 123006 (2016).

5. S. T. Park, M. M. Lin, and A. H. Zewail, New J. Phys. 12, 123028 (2010).

6. F. J. G. de Abajo, A. Asenjo-Garcia, and M. Kociak, Nano Lett. 10, 1859 (2010). 